\title{
Les Jacobins de province et Paris : le cas franc- comtois
}

Danièle Pingué

\section{(2) OpenEdition \\ 12 Journals}

Édition électronique

URL : https://journals.openedition.org/ahrf/770

DOI : 10.4000/ahrf.770

ISSN : 1952-403X

Éditeur :

Armand Colin, Société des études robespierristes

\section{Édition imprimée}

Date de publication : 1 décembre 2002

Pagination : 101-113

ISSN : 0003-4436

\section{Référence électronique}

Danièle Pingué, "Les Jacobins de province et Paris : le cas franc-comtois », Annales historiques de la Révolution française [En ligne], 330 | octobre-décembre 2002, mis en ligne le 17 avril 2008, consulté le 22 avril 2022. URL : http://journals.openedition.org/ahrf/770 ; DOI : https://doi.org/10.4000/ahrf.770 


\title{
LES JACOBINS DE PROVINCE ET PARIS : LE CAS FRANC-COMTOIS
}

\author{
DANIÈLE PINGUÉ
}

\begin{abstract}
Relativement peu nombreuses, fonctionnant dans un environnement plutôt difficile, les sociétés franc-comtoises sont, par contre, fortement intégrées au réseau constitué par le Club des jacobins de Paris et ses filiales. Dans ce cadre, elles ont des relations très denses avec la capitale. Par le canal de leur correspondance " verticale " avec la société mère, elles diffusent dans leur cidevant province des informations, des directives, des modèles de comportement émanant de Paris, mais elles sont en même temps les voix de la province vers la capitale. Leurs relations " horizontales " avec les filiales des autres régions favorisent également leurs relations avec Paris et plus largement, l'intégration de la région à l'espace national.
\end{abstract}

Mots clés : Franche-Comté ; Jacobins ; sociétés politiques.

Dans les rapports province-Paris/Paris-province au cours de la Révolution, les sociétés politiques ont joué un rôle de premier plan. Elles étaient en contact régulier avec la capitale par de nombreux canaux : les journaux, dont la lecture fut l'une de leurs toutes premières raisons d'être, leur correspondance avec les députés ou d'autres citoyens de leur région présents à Paris, leurs relations avec le Club des Jacobins, puis, surtout lorsqu'elles furent devenues des organes quasi officiels, leurs multiples liens avec le gouvernement (qu'il s'agisse des directives qu'elles recevaient des autorités centrales, de leurs rapports privilégiés avec les représentants en mission ou encore, des adresses qu'elles envoyaient à la Convention...); par tous ces canaux, elles recevaient de Paris des informations et des directives, mais elles pouvaient également faire parvenir dans la capitale la voix des provinces.

Naturellement, la densité, la forme, le contenu de ces échanges ont varié, en fonction, notamment, du contexte géopolitique (1). Par exemple,

(1) Voir en particulier : Jean BOUTIER et Philippe BOUTrY (dir.), Atlas de la Révolution française, fasc. 6: Les sociétés politiques, Éd. de l'EHESS, Paris, 1992 ; Michel VoVELLE, La découverte de la politique, 
dans le cadre de la Haute-Normandie, proche de Paris, riche en clubs, plutôt favorable à la Révolution, ils furent très abondants et se déroulèrent réellement dans les deux sens (2). Le présent article aborde le cas d'une région très différente de la précédente, la Franche-Comté, et porte sur l'un des aspects les plus originaux, nous semble-t-il, des rapports des clubs de cette ancienne province avec la capitale : leurs relations avec Paris en raison de leur appartenance au «réseau jacobin». Il repose sur deux types de sources : le recueil de documents publié par Alphonse Aulard (3), et les registres de délibérations des associations (4).

Dans son règlement du 8 février 1790, la "Société des Amis de la Constitution séant aux Jacobins » affirmait sa vocation de constituer «un centre commun " pour les sociétés « du même genre " établies en France, qui choisiraient de devenir ses filiales. Avec celles-ci, s'établirait une correspondance décrite ainsi : «elle recevra leurs instructions et leur transmettra les vues qui résulteront du rapprochement des Lumières et des intérêts » (5) ; il s'agissait donc d'une correspondance prévue dans les deux sens - Paris-province, mais aussi province-Paris -, pour laquelle le club parisien se dota d'un puissant comité de correspondance. Par ailleurs, se développa entre les filiales une correspondance «horizontale » qui ne fut pas sans incidences sur leurs relations avec la capitale.

Quels furent la densité, la teneur et, s'il est possible de les mesurer, les résultats de ces échanges pour les sociétés de Franche-Comté? Pour répondre à ces questions, il convient tout d'abord de présenter l'environnement géopolitique de ces clubs, ainsi que les caractéristiques de leur réseau. Nous examinerons ensuite leurs rapports «verticaux» avec la société mère avant de nous intéresser brièvement, pour terminer, au rôle qu'ont pu jouer leurs relations « horizontales » avec les clubs d'autres régions.

Compte tenu de l'état des sources - les registres contemporains de la Terreur ayant fait l'objet de nombreuses destructions - notre propos portera principalement sur les années 1790-1793.

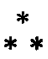

géopolitique de la Révolution française, Paris, La Découverte, 1993; Christine PeYraRd, Les Jacobins de l'Ouest, Paris, Publications de la Sorbonne, 1996.

(2) Danièle PINGUÉ, Les mouvements jacobins en Normandie orientale, Paris, Éd. du CTHS, 2001.

(3) F.A. AULARD, La société des Jacobins, recueil de documents sur l'histoire du club des Jacobins de Paris, Paris, Cerf, 1889-1897, 6 volumes.

(4) Ont été consultés les registres des sociétés de Gray et Jussey (Haute-Saône), Saint-Hippolyte, Ornans et Belvoir (Doubs), Lons-le-Saunier (Jura).

(5) F.-A. AULARD, La société des Jacobins..., op. cit., t. I, p. XXIX. 
L'ancienne province de Franche-Comté - récemment annexée, en 1678 par le traité de Nimègue -, a été divisée, au début de la Révolution, en trois départements : le Doubs, la Haute-Saône et le Jura (6) .

Sur le plan géographique, elle présente alors des conditions plutôt défavorables aux relations avec la capitale. Malgré de grands progrès au XVIIIe siècle, Besançon reste à quatre jours de route de Paris et continue à donner, en 1789, une impression d'isolement. Arthur Young note le 27 juillet : « [...] Dans toute la ville de Besançon, pas de trace du Journal de Paris ou d'un autre journal donnant le détail des délibérations des états; et cependant, c'est la capitale d'une province [...] et, chose étrange à dire, le courrier n'arrive que trois fois par semaine ! [...] Je n'aime pas l'air et les manières des gens d'ici, et je voudrais voir Besançon englouti par un tremblement de terre, avant que je fusse contraint à y vivre [...] » (7). La population de chacun des départements est assez faible - entre 200000 et 300000 habitants, le Doubs étant d'ailleurs le moins peuplé - et Besançon, avec 25000 habitants, est la seule ville importante. De plus, le relief et le climat rendent la circulation intérieure difficile (on sait que Mouthe est considérée encore aujourd'hui comme la "petite Sibérie française"). Nous sommes loin du cas évoqué plus haut de la Haute-Normandie, proche de Paris, aisément accessible, très peuplée et très urbanisée. Néanmoins, la FrancheComté possède avec cette dernière un point commun: celui d'être limitrophe de la frontière. Cette situation alimente chez ses habitants un réel sentiment patriotique et lui donne, aux yeux du gouvernement, une grande importance politique et stratégique.

En ce qui concerne «l'esprit public», selon l'historiographie du XIXe siècle, la région aurait constitué en bloc une zoné de résistance à la Révolution. En réalité, il faut établir des distinctions dans l'espace et dans le temps. Besançon et d'autres villes, comme Gray (Haute-Saône) ou Dole (Jura), à la pointe du combat révolutionnaire, s'opposent aux campagnes difficilement pénétrées par les idées nouvelles. D'autre part, jusqu'au printemps 1793, les Francs-Comtois, malgré leur hostilité à la Constitution civile du clergé, adhèrent assez volontiers à la Révolution, comme le montrent, notamment, les forts taux de participation électorale (8). Par contre, à partir de l'été 1793 , ils vont déployer, face à la politique des Montagnards, une véritable résistance qui n'ira pas, toutefois, jusqu'à la lutte armée.

(6) Belfort faisait alors partie du Haut-Rhin et ne sera pas concerné par cette étude.

(7) Arthur Young, Voyages en France en 1787, 1788 et 1789, Traduction par Henri Sée, Paris, A. Colin, 1931, 3 tomes.

(8) Cf. Danièle PINGUÉ, «L'engagement associatif et la mobilisation électorale dans les premières années de la Révolution : des pratiques complémentaires ou alternatives? ", dans La Révolution française : idéaux, singularités, influences, Actes des journées d'étude de Vizille, 20-21 septembre 2001, Grenoble, Presses Universitaires de Grenoble, 2002. 
Dans les premières années de la Révolution, dans un contexte politique qui leur est donc plutôt favorable, les sociétés se développent à un rythme soutenu. À la veille de l'an II, on en compte au moins 24 dans le Doubs, 24 dans le Jura et 11 en Haute-Saône, ce qui représente une densité supérieure à la moyenne pour la France septentrionale (9), et aucun district de la région n'échappe à leur influence. De plus, en général, ces associations rayonnent sur plusieurs communes et pratiquent un recrutement massif. Ainsi, celle de Gray, chef-lieu de district d'environ 6000 habitants, rassemble près de 800 citoyens, dont un quart, extérieurs à la commune, résident dans 61 localités différentes réparties dans l'ensemble des cantons des deux districts de Gray et Champlitte (10). Enfin, toutes ces sociétés, comme celles des autres régions, sont unies par de nombreux liens, et constituent un véritable réseau solidement relié, comme nous allons le voir, au Club des Jacobins de Paris.

Par contre, en l'an II, comme tous ceux du Nord-Est, les départements francs-comtois restent relativement à l'écart de «l'explosion généralisée de la sociabilité politique révolutionnaire" (Boutier, Boutry) observée à l'échelle nationale. Aussi, au début de l'été 1794, par rapport à celle d'autres régions, la densité de leur réseau associatif est devenue faible, avec seulement 55 communes dotées d'un club dans le Jura, 49 en Haute-Saône et 47 dans le Doubs (11). De surcroît, ces associations se trouvent désormais sauf exception - dans un environnement plutôt hostile et réagissent en développant une surenchère ultra-révolutionnaire, ce qui contribue à les isoler encore davantage.

Au total, sur l'ensemble de la période 1790-1793, les sociétés franccomtoises constituent donc un réseau modeste, fonctionnant dans un environnement contrasté. Mais cette fragilité ne les empêche pas d'être étroitement liées au réseau national des Jacobins.

Tout d'abord, on est frappé par l'importance que les grands clubs francs-comtois accordent à leur correspondance avec la capitale.

Celui de Besançon, qui va dominer sans conteste le réseau régional, en fait même au départ sa principale raison d'être. En août 1790, les membres fondateurs informent la municipalité qu'ils ont l'intention de se réunir régulièrement "pour faire soit à l'Assemblée nationale, soit au Roy, soit aux corps administratifs et municipaux toutes les pétitions qu'ils croiront utiles

(9) Au nord de la ligne La Rochelle -Genève, la moyenne est alors de 16 sociétés par département.

(10) AD Haute-Saône, 361 L 3, société populaire de Gray.

(11) Sources : Jean Boumer et Philippe BOUTRY (dir.), Atlas de la Révolution française..., op. cit. et Colette MERLIN et Henti HOURS, Les sociétés populaires du Jura pendant la Révolution, choix de documents, Arch. dép. du Jura, 1990; à ces inventaires s'ajoutent quelques sociétés que nous avons « découvertes " par la suite, ce qui ne modifie en rien les ordres de grandeur. La moyenne nationale est alors de 62 sociétés par département. 
au bien public et au maintien de la Constitution" (12). Le nouveau club obtient immédiatement, semble-t-il, son affiliation à celui des Jacobins de Paris (13), s'empresse d'en adopter le règlement et noue rapidement des relations horizontales avec les autres filiales (14). Par la suite, appelé à conseiller des «sociétés naissantes», c'est sur l'importance du réseau jacobin et l'intérêt qu'il y aurait à le rejoindre, qu'il insiste en priorité. Ainsi, au club d'Ornans qui le contacte en janvier 1791, il adresse quatre exemplaires de son règlement, un discours de Robespierre, le numéro Un du Journal des Amis de la Constitution (15) et une description dithyrambique des Sociétés des Amis de la Constitution, présentées comme un « [...] nombre infini d'associations [regroupant] les meilleurs patriotes de toutes les villes du royaume, réunis ensemble et ne faisant qu'un seul tout par le centre commun auquel tous aboutissent "(16). Ce grand intérêt du club de Besançon pour sa correspondance avec Paris persiste au-delà des premières années de la Révolution, comme le soulignent les articles XI et XII de son nouveau règlement, du 28 floréal an II (11 mai 1794) : "Aucune adresse, circulaire et lettre pour Paris ne partent sans avoir été approuvées par la société. Trois membres de ce comité (de correspondance) sont chargés en particulier et sous leur responsabilité, de la correspondance avec Paris » (17).

Les sociétaires de Lons le Saunier se montrent très soucieux, eux aussi, de leur correspondance avec la capitale et plus particulièrement avec les Jacobins. En janvier 1791, alors que le chef-lieu du Jura abrite deux sociétés rivales, c'est l'acharnement de l'une et l'autre à revendiquer son affiliation à la société parisienne qui amène celle-ci à prendre un arrêté lourd de conséquences : celui de n'accorder qu'une seule affiliation par commune (18).

(12) AM Besançon, BB 204, Délibérations municipales, séance du 28 août 1790.

(13) Le club de Besançon figure sur la liste des 91 sociétés alors affiliées aux Jacobins de Paris, publiée dans Le Patriote Français le 15 août 1790 (BNF, $4^{\circ} \mathbf{L c} 185$ ).

(14) $\grave{A}$ une lettre de 80 citoyens sollicitant leur adhésion, datée du 13 septembre 1790 , il adresse la réponse suivante : "Il existe parmi nous [...] un règlement particulier pour l'admission des récipendiaires. Ce règlement a été adopté par notre sociêté et par nombre d'autres répandues dans les différentes villes du royaume, avec lesquelles nous avons correspondance " (BM Besançon, Ms 241 055, Société populaire).

(15) Un arrêté du 31 octobre 1790, pris par la Société des Amis de la Constitution de Paris, autorise un de ses membres, Choderlos de Laclos, à publier périodiquement la «correspondance des Amis de la Constitution »; le Journal des Amis de la Constitution, créé à cet effet, voit le jour le 30 novembre 1790 et va publier 41 numéros, jusqu'à sa disparition le 20 septembre 1791.

(16) AD Doubs, L 2360, société populaire d'Omans, séance du 7 février 1791 (transcription d'une lettre de la Société des Amis de la Constitution de Besançon, signée Michaud, président, Laurent, Gaye et Janson, secrétaires).

(17) BM Besançon, Ms 241 056, Société populaire.

(18) Le «Comité de l'Arrosoir » fondé en juin 1790 et devenu la Société des Amis de la Constitution bénéficiait déjà de cette affiliation ; en décembre, se forma sur l'initiative de Théodore Lameth, frère des deux célèbres Constituants et président de l'administration départementale, une société concurrente qui sollicita à son tour l'affiliation mais qui fut dénoncée par la précédante dans un article publié dans Les révolutions de France et de Brabant de Camille Desmoulins. C'est au spectacle de cette polémique d'ampleur nationale que les Jacobins prirent leur arrêté, au profit, d'ailleurs du club de Théodore Lameth. Les deux sociétés finirent par fusionner en mai 1791 (Sources : F.A. AULARD, La société des Jacobins..., op. cit., t. II, p. 62 et H. LiBoIS, « Délibérations de la Société populaire de Lons-le-Saunier, du 5 novembre 1791 au 
Dans la société résultant de la fusion des deux précédentes, en mai 1791, la majorité des débats vont être liés aux rapports avec la société mère. Signe de la priorité qu'elle accorde à la correspondance, cette association modifie au moins quatre fois le calendrier de ses réunions afin de le faire correspondre aux « jours de la poste » (19) .

À l'exemple des clubs leaders de la région, jusqu'à la chute de la monarchie, la majorité des sociétés franc-comtoises sollicitent dès leur fondation leur affiliation au Club des Jacobins de Paris. Pour cela, il leur suffit de bénéficier du parrainage de deux clubs déjà affiliés et d'accompagner leur demande de la liste de leurs membres. Dans l'ensemble, elles obtiennent aisément satisfaction (20). Le club de Gray constitue la seule exception, mais l'acharnement qu'il met pour obtenir finalement gain de cause montre l'importance de sa motivation. Ayant demandé une première fois cette affiliation le jour même de sa création, le 17 avril 1791, il est d'abord invité à rechercher le parrainage de deux sociétés, que celles de Besançon et de Dole lui accordent sans peine, le 9 juin; mais la fuite du roi, puis la scission des Feuillants retardent la réponse de la société mère qui finit par demander le 8 septembre une nouvelle attestation de parrainage; après une longue hésitation - un ancien leader du club devenu député à l'Assemblée législative, Jean-François Crestin, vient de se rallier aux Feuillants - les Jacobins de Paris finissent par accorder l'affiliation tant convoitée le 29 décembre 1791 (21). Au total, à la veille du 10 août 1792, sur les 40 sociétés dont la présence est attestée en Franche-Comté, 25 au moins - plus de la moitié - sont affiliées au club parisien (22) ; si la majorité d'entre elles siègent dans un chef-lieu de district (23) ou de canton (24), trois - fait assez remarquable - se situent dans une simple commune (25).

Après la chute de la Monarchie, la société mère devient plus réticente, mettant en doute la pureté des sociétés récentes et n'accorde plus son affiliation qu'au compte-gouttes. Sur les 32 sociétés franc-comtoises apparues d'août 1792 à septembre 1793, à notre connaissance, quatre seulement

25 juin 1793 ", Société d'Émulation du Jura, 6e série, 1, 1895-1896, Appendice, p. 209 ; les registres de délibérations pour la période concernée ont malheureusement disparu).

(19) Le 5 novembre 1791, le 20 janvier et le 8 juillet 1792, le 28 janvier 1793 (H. LiBoIS, op. cit.).

(20) Le club d'Ornans, qui sollicite cette affiliation le 10 février 1791 l'obtient le 21 avril; celui de Quingey, qui la sollicite le 7 mars 1792 l'obtient le 1 er avril (AD Doubs, L 2360, Société populaire d'Omans et L 2362, Société populaire de Quingey).

(21) AD Haute-Saône, 361 L 1, Société populaire de Gray.

(22) Faute de listes des filiales du club parisien postérieures au 19 juin 1791, on n'a aucune certitude au sujet des autres - sauf si on possède leur registre - mais rien ne prouve qu'elles ne sont pas dans le même cas.

(23) Besançon, Baume, Ornans, Pontarlier, Quingey (Doubs), Lons-le-Saunier, Salins, Dole, SaintClaude (Jura), Vesoul, Champlitte, Gray, Lure et Luxeuil (Haute-Saône).

(24) Recologne, Jougne, Morteau (Doubs), Conliège, Clairvaux, Champagnole (Jura), Favernay et Marnay (Haute-Saône).

(25) Serre-et-Frasnois, Chantrans et Valdahon (Doubs). 
l'obtiennent (26). Par exemple, celle de Saint-Hippolyte, chef-lieu de district du Doubs, la sollicite sans succès. Mais cela n'empêche pas un certain nombre de non affiliées de se référer explicitement au réseau jacobin. Le premier président de la société de Jussey, chef-lieu de district de HauteSaône, créée le 6 avril 1793, rend hommage dans son discours inaugural à "la société populaire de Paris, mère de toutes les sociétés de la République » (27). La société de Belvoir, dans le Doubs, désire, selon son règlement du 17 février 1793 «s'associer aux autres sociétés du même genre $»(28)$.

En l'an II, l'extrême méfiance de la société mère se traduit en FrancheComté par une absence totale de nouvelles affiliations ayant laissé une trace dans les archives. Cependant, compte tenu de la faible densité des clubs, la proportion des sociétés affiliées à celle de Paris ( 29 sur 151 , soit près d'une sur cinq) est relativement élevée. De plus, neuf sociétés de la région, pour la plupart non affiliées, sont abonnées à un moment donné au Journal de la Montagne, organe de presse quasi officiel des Jacobins de mai 1793 à novembre 1794 (29).

Autre indice de leur solide ancrage dans le réseau jacobin, à l'occasion des crises que celui-ci traverse au cours des premières années de la Révolution, les sociétés franc-comtoises se placent résolument sur les positions de la société mère.

La crise des Feuillants, qui met en péril l'existence même du réseau au cours de l'été 1791, constitue un premier test. Sur les 23 sociétés alors recensées en Franche-Comté, neuf sont affiliées à celle de Paris (30) et quatre au moins en ont fait récemment la demande (31). Au moins sept d'entre elles - Besançon, Ornans, Pontarlier, Vesoul, Dole, Saint-Claude, Baume -, « écrivent sur la scission » à la société mère; seul le club de Saint-Claude " exprime des sentiments divers », tandis que les Bisontins et des Vésuliens «jurent un attachement inviolable et désirent la réunion »; mais tous se rallient finalement au Club des Jacobins (32). Leurs registres de délibérations permettent de mieux connaître la réaction de deux sociétés. À Ornans, le 21 juillet, à la réception de la lettre des Feuillants datée du 16 qui annonce la scission, «[...] cette nouvelle », note le secrétaire, «a affligé tous les membres composant la

(26) Pont-de-Roide (Doubs), Arbois, Orgelet (Jura), Pesmes (Haute-Saône)

(27) AD Haute-Saône, 363 L 1, Société populaire de Jussey.

(28) AD Doubs, L 2352, Société populaire de Belvoir.

(29) Les sociétés de Frasne et L'Isle sur le Doubs (Doubs), Gigny, Saint-Claude, Clervaux-les-Lacs et Salins (Jura), Vesoul, Dampierre-sur-Salon et Luxeuil (Haute-Saône) (AN, T 1495 A et B).

(30) Besançon, Ornans, Pontarlier (Doubs), Lons-le-Saunier, Salins, Dole, Saint-Claude (Jura), Vesoul, Luxeuil (Haute-Saône)

(31) Recologne, Baume (Doubs), Favernay, Gray (Haute-Saône) ; les sociétés de Lure (HauteSaône), Conliège, Clairvaux, Champagnole (Jura) apparues au printemps 1791 seront par la suite affiliées au Club des Jacobins; on ignore si à cette date, elles en ont déjà fait la demande.

(32) F.-A. AULARD, La société des Jacobins..., op. cit., t. III, pp. 66-92. 
société [...] qui sont persuadés qu'il ne pouvait rien arriver de plus fâcheux dans les moments critiques que la division parmi mes patriotes ». La décision est prise d'attendre de plus amples informations qui parviennent, trois jours plus tard, sous la forme de l'adresse de la "Société des Amis de la Constitution séant aux Jacobins », du 17 juillet, signée Dufourny. Le premier mouvement du club d'Ornans est alors « d'écrire aux deux sections pour les prier de se réunir », mais il continue de s'interroger, à la lumière notamment d'adresses reçues de diverses sociétés - Dijon, qui plaide, elle aussi pour la réunion des deux camps, Tours qui choisit les Feuillants, Versailles qui se rallie aux Jacobins - et, dès le 4 août, opte pour les Jacobins « restés fidèles à la Constitution et à la liberté ». Cette décision rapide est en même temps ferme et définitive : les nouvelles adresses de sociétés favorables à la réconciliation - Marseille, Saint-Germain-en-Laye, Bordeaux - sont déclarées sans objet; le 28 août, l'ouvrage relatif à l'élection des députés à l'Assemblée législative - Opinion énoncée dans l'Assemblée des Amis de la Constitution séante aux Feuillants sur le mode de convocation des assemblées constituantes, par Louis Ramond, membre de cette société - est «brûlé au milieu de la salle d'assemblée comme contenant des principes absurdes, révoltants et totalement anticonstitutionnels" et «la Société séante aux Jacobins" sera informée de la diffusion de cet écrit (33). Pour sa part, la société de Gray, en cours d'affiliation, se demande à qui elle va désormais s'adresser, mais décide, pratiquement sans débat, de choisir les Jacobins « car on connaît bien cette société qui est patriotique " (34).

Bien que d'une ampleur sans commune mesure avec la crise précédante, l'affaire Le Chapelier suscite quelques remous à l'intérieur du réseau jacobin au cours de l'hiver 1791-1792. Il semble que la loi des 29 et 30 septembre 1791, votée sur l'initiative du député breton, combattue notamment par Robespierre à l'Assemblée, ait été diversement appréciée dans les clubs de province. Toujours est-il que leur auteur, de retour dans l'Ouest après la séparation de l'Assemblée constituante, se voit exclu du Club national de Bordeaux, tandis que son admission provoque une scission au sein de la Société des Amis de la Constitution de Rennes (35). Les deux associations concernées informent de la situation les autres filiales du Club des Jacobins de Paris. On est néanmoins surpris de constater que deux sociétés de Franche-Comté, au moins, participent activement à ce débat : celle d'Ornans rompt le 26 janvier 1792 toute correspondance avec la société de Rennes qui a admis Le Chapelier; le club de Lons-le-Saunier fait de même le 5 février :

(33) AD Doubs, L 2360, Société populaire d'Omans, séances du 21 et 24 juillet, 4, 18 et 28 août 1791.

(34) AD Haute-Saône, 361 L 1, Société populaire de Gray, séance du 31 juillet 1791.

(35) Selon les informations fournies par les registres de la société d’Ornans, séances du 10 novembre et 15 décembre 1791 et de Lons-le-Saunier, séances du 3 et 5 février 1792. 
"cet ennemi du peuple français », explique-t-il, « avait encouru notre haine et notre mépris par sa conduite inconstitutionnelle contre l'établissement des sociétés populaires » (36).

Enfin, en juin 1793, alors que l'administration départementale du Jura a rompu avec la Convention devenue montagnarde et que celles du Doubs et de la Haute-Saône sont hésitantes, les sociétés politiques se transforment en véritables pôles de résistance anti-fédéraliste. Celle de Gray va d'ailleurs s'intituler, à partir du 15 août, « Société républicaine, montagnarde et antifédéraliste». Contrairement aux clubs d'autres régions touchées par la « révolte», comme la Basse-Normandie, dont un grand nombre rompent alors leur affiliation à la société mère, les sociétés franc-comtoises se trouvent une fois de plus en parfait accord avec les Jacobins de Paris.

Dans leurs rapports avec la capitale, leurs relations « verticales » avec la société mère jouent naturellement un rôle primordial. Examinons d'abord celles qui ont lieu dans le sens Paris-province.

On peut avoir une idée assez précise des «papiers » émanant de Paris, grâce au recueil des documents publiés par Alphonse Aulard, qui présente une soixantaine de circulaires expédiées par le Club des Jacobins à ses filiales entre août 1790 et juin 1793. On peut les classer en quatre catégories : des demandes d'information, des informations accompagnées de commentaires, de véritables directives et enfin, des mesures concernant le développement et l'organisation du réseau. À ces circulaires s'ajoutent de nombreux documents bruts (discours, textes de lois...) ainsi que la correspondance personnalisée adressée à une société en particulier.

Les registres des clubs de Franche-Comté mentionnent abondamment ce courrier, montrant par là qu'ils lui accordent une grande importance. Une comparaison attentive avec le recueil d'Alphonse Aulard permettrait d'ailleurs de répertorier les documents que celui-ci ne cite pas. Ainsi, le registre de la société d'Ornans, d'avril 1791 à septembre 1793, fait état pratiquement à chaque séance de lettres ou de «paquets» de la société mère. Contrairement à ce qu'on peut constater à Rouen, par exemple, ou dans les sociétés de Basse-Normandie étudiées par Christine Peyrard (Lisieux), ces documents représentent ici la majorité du courrier reçu.

Quels sont les impacts de cette correspondance?

Tout d'abord, indéniablement, les Francs-Comtois copient les pratiques du club parisien. La société de Besançon, on l'a vu, en a adopté le règle-

(36) $\mathrm{AD}$ Doubs, L 2360, Société populaire d'Ornans, séance du 26 janvier $1792 ; \mathrm{H}$. LIBOIS, “Délibérations de la Société populaire de Lons... ", op. cit., séance du 5 février 1792. 
ment. Comme par la suite, les sociétés d'Ornans, en janvier 1791, puis de Quingey, en mars 1792 - deux chefs-lieux de districts -, adoptent à leur tour le règlement de Besançon, on peut dire que le modèle est largement diffusé! On ne constate pas le même phénomène en Haute-Normandie où l'on ne trouve jamais deux règlements identiques. Autres exemples, le 27 mars 1791, un membre du club d'Ornans propose d'adopter le serment des Jacobins de Paris, reproduit, précise-t-il, «dans le Journal des Amis de la Constitution, $\mathrm{n}^{\circ} 13$, p. 492 "; le 17 novembre, un autre membre propose d'établir des billets d'entrée "ainsi qu'on le pratique dans la société mère, dans celle de Besançon et dans presque toutes les sociétés de l'Empire».

Les documents fournis par la société mère permettent également aux clubs de bien connaître les éléments des débats sur les grands problèmes nationaux. Ainsi, la société d'Ornans, en novembre 1792, discute du sort du roi en reprenant tous les arguments avancés à la Convention; le débat est tellement animé que les intervenants sont invités à rédiger à l'avance leur discours et à le déposer sur le bureau.

Les demandes de renseignements émanant de Paris donnent généralement lieu à des réponses, sur lesquelles nous reviendrons, à propos du courrier échangé dans le sens Province-Paris.

Enfin, on peut se demander quels sont les résultats des directives émanant de la société mère. Prenons l'exemple de celles qui concernent les élections. Entre 1790 et 1792, les Jacobins de Paris émettent au moins quatre circulaires sur ce thème : en octobre 1790 , sur la nécessité de faire un bon choix pour l'élection des juges de paix; en juin et à nouveau en juillet 1791, sur la nécessité de participer aux assemblées primaires et de faire les bons choix dans les élections à l'Assemblée législative; enfin, le 22 août 1792, sur la nécessité de bien choisir les députés à la Convention. Les circulaires de 1790 et 1791 insistent sur la nécessité de se rendre aux assemblées primaires, et dressent le profil de l'élu idéal, qui doit se distinguer par son talent mais surtout par sa vertu. Celle du 22 août 1792 va beaucoup plus loin : dans le but d'éviter à tout prix la réélection des Feuillants, elle désigne nommément 224 députés de l'Assemblée législative (ceux qui ont voté pour le décret d'accusation de La Fayette) et 40 anciens constituants (dont Robespierre, Pétion, Buzot, etc.) qu'elle propose au suffrage des électeurs; par la même occasion, sont donc désignés les législateurs et les constituants qui ne doivent pas être réélus. En même temps, ce texte annonce l'élargissement du suffrage : «Elle [l'Assemblée] a établi le règne de l'égalité en appelant sans distinction le peuple à l'exercice de sa souveraineté [...]. Les électeurs étant payés à trois livres par jour et à 20 sols par lieue pour leur voyage, il n'est plus nécessaire d'être riche bourgeois, prêtre ou ci-devant noble pour accepter cette noble mission. » 
Les échos de ces circulaires sont présents dans les registres des clubs et les directives qu'elles contiennent sont toujours suivies d'effet. En particulier, les sociétés franc-comtoises se mobilisent à fond en faveur des élections à la Convention. Ainsi, le 18 août, celle de Lons-le-Saunier décide de se réunir chaque jour « pour discuter au sujet des élections qui doivent se faire pour la Convention nationale »; le 22 août, elle décrète que «les membres qui n'iront pas voter seront expulsés de la société et rayés du tableau des membres »; le dimanche 25 , «plusieurs membres ont fait et lu des discours pour inviter les personnes de la campagne présentes à la séance de faire le bon choix dans les élections qui se préparent pour la Convention nationale » et le président lit à cette occasion la circulaire des Jacobins de Paris. À Gray, le 2 septembre, les électeurs sont invités à la séance du club et un membre fait un discours pour " démontrer l'indispensable nécessité de faire choix de citoyens justes et éclairés pour la formation de la Convention nationale ». Cet investissement en matière électorale porte d'ailleurs ses fruits. En 1791, cinq des six élus du Doubs à l'Assemblée législative sont membres du club de Besançon; sont également élus, par exemple, deux membres du club de Gray, un membre du club de Champlitte, Haute-Saône, etc. En 1792, aucun des députés Feuillants de Haute-Saône et du Jura n'est réélu à la Convention.

Examinons à présent les relations des clubs avec la société mère dans le sens province-Paris.

Les registres extrêmement bien tenus du club d'Ornans offrent un exemple précis de cette correspondance. Du 15 mai 1791 (l'affiliation a été obtenue le 25 avril) au 20 septembre 1793, les procès-verbaux font état de huit lettres adressées au Club des Jacobins, dont le contenu est le suivant :

- 15 mai 1791 : demande que le roi, en tant que chef des armées, porte à son chapeau un bouton où sera inscrit «à la Nation ";

- 28 août 1791 : récit de la réception d'une adresse des Feuillants, qui a été brûlée;

- 20 septembre 1791 : satisfaction que le roi ait accepté la Constitution (doubles adressés au roi et à l'Assemblée);

- 15 décembre 1791 : appui à la société mère dans ses revendications à propos du veto royal; en même temps, renseignements précis sur la résistance religieuse dans le district d'Ornans;

- 9 février 1792 : demande que la Nation s'allie aux Anglais, Polonais et Américains;

- 17 avril 1792 : contenu non précisé (adressé également à l'Assemblée);

- 19 août 1792 : demande de faire supprimer une tapisserie de la galerie du château de Versailles représentant «le gouverneur de Dole remettant à genoux les clefs de la ville au despote Louis XIV »; 
- 25 août 1792 : adhésion aux décrets du 10 août (le même jour, adresse à l'Assemblée... pour se plaindre de retard dans le courrier);

- 30 août 1792 : demande d'armes pour les volontaires de la région (copie adressée au ministre de la Guerre);

-27 juillet 1793 : récit de l'acceptation privée, dans le cadre de la société, de la Constitution (une adresse à la Convention sur le même thème est seulement envoyée le 11 août).

Au total, deux de ces lettres contiennent des récits, trois consistent en des déclarations d'adhésion à des positions politiques du club parisien, auxquelles peuvent être jointes des réponses à des demandes d'informations, deux sont des revendications de politique générale, deux enfin, des revendications politiques locales. Près de la moitié donc porte sur des revendications : la voix de la province qui s'exprime ici ne se limite pas à de simples déclarations d'adhésion à la politique "parisienne ». Il est intéressant par ailleurs de constater que c'est en priorité à la société mère que le club d'Ornans adresse des textes relevant plutôt de la compétence de l'Assemblée nationale. En Haute-Normandie, par exemple, c'est presque exclusivement à l'Assemblée que les sociétés adressent ce type de missives. Le fait qu'il n'en aille pas de même ici témoigne, à côté des indices déjà évoqués, de l'importance des liens des clubs francs-comtois avec la société parisienne.

La correspondance horizontale avec les filiales des différentes régions a elle-même un impact sur les relations des clubs avec la capitale. Il n'est pas rare en effet qu'une société appuie une proposition émanant d'une autre filiale en envoyant elle-même à l'Assemblée nationale une adresse de la même teneur. Ainsi, à la réception d'une lettre de la Société des Amis de la Liberté et de l'Égalité de Nevers demandant que les fonctions inamovibles - receveurs de l'enregistrement, ingénieurs, notaires...- deviennent électives, la société de Lons-le-Saunier « a délibéré qu'elle adhérait [...] à la pétition faite pas les citoyens de Nevers et qu'elle le manifestait par une pétition à la Convention » (37). Autre exemple, le 28 fructidor an II (14 septembre 1794), la société de Quingey, après avoir pris connaissance d'une adresse de celle d'Auxerre, dont elle ne précise pas le contenu, rédige un projet d'adresse à la Convention réclamant la prompte organisation des comités révolutionnaires de district « pour donner une nouvelle vie à l'esprit public et un coup de mort à tous les ennemis déclarés ou secrets de l'égalité et de la liberté " (38).

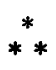

(37) Ibid, séance du 10 janvier 1793.

(38) AD Doubs, L 2362, Société populaire de Quingey, séance du 28 fructidor an II. 
Il ressort de cette brève étude que les sociétés politiques de FrancheComté, relativement peu nombreuses et amenées à fonctionner, à partir de l'an II, dans un contexte plutôt difficile, étaient par contre bien intégrées au réseau national des Jacobins. Dans ce cadre en particulier, elles ont développé des relations très denses avec la capitale. À côté d'autres agents, elles ont ainsi contribué à faire de la Révolution une étape essentielle du processus d'intégration de la Franche-Comté à la France.

\author{
Danièle PINGUÉ \\ Laboratoire des sciences historiques \\ Université de Besançon \\ 23, rue Louis Monnier, « Les Pierres Vives » \\ 70000 Vesoul \\ daniele.pingue@wanadoo.fr
}

